\title{
Probing TARP Modulation of AMPA Receptor Conductance with Polyamine Toxins
}

\author{
Alexander C. Jackson, ${ }^{1 \star}$ Aaron D. Milstein, ${ }^{1,2,3 \star}$ David Soto, ${ }^{4 \star}$ Mark Farrant, ${ }^{4 * \star}$ Stuart G. Cull-Candy, ${ }^{4 * \star}$ \\ and Roger A. Nicoll ${ }^{1,2,3 * *}$ \\ Departments of ${ }^{1}$ Cellular and Molecular Pharmacology and ${ }^{2}$ Physiology and ${ }^{3}$ Neuroscience Graduate Program, University of California, San Francisco, \\ California 94143, and ${ }^{4}$ Department of Neuroscience, Physiology, and Pharmacology, University College London, London WC1E 6BT, United Kingdom
}

The properties of synaptic AMPA receptors (AMPARs) depend on their subunit composition and association with transmembrane AMPAR regulatory proteins (TARPs). Although both GluA2 incorporation and TARP association have been shown to influence AMPAR channel conductance, the manner in which different TARPs modulate the mean channel conductance of GluA2-containing AMPARs is unknown. Using ultrafast agonist application and nonstationary fluctuation analysis, we found that TARP subtypes differentially increase the mean channel conductance, but not the peak open probability, of recombinant GluA2-containing AMPARs. TARP $\gamma$-8, in particular, enhances mean channel conductance to a greater degree than $\gamma-2, \gamma-3$, or $\gamma-4$. We then examined the action of a use-dependent antagonist of GluA2-containing AMPARs, philanthotoxin-74 (PhTx-74), on recombinant AMPARs and on GluA2containing AMPARs in cerebellar granule neurons from stargazer mice transfected with TARPs. We found that the rate and extent of channel block varies with TARP subtype, in a manner that correlates linearly with mean channel conductance. Furthermore, block of GluA2-containing AMPARs by polyamine toxins varied depending on whether channels were activated by the full agonist glutamate or the partial agonist kainate, consistent with conductance state-dependent block. Block of GluA2-lacking AMPARs by $\mathrm{PhTx}-433$ is also modulated by TARP association and is a function of agonist efficacy. Our data indicate that channel block by polyamine toxins is sensitive to the mean channel conductance of AMPARs, which varies with TARP subtype and agonist efficacy. Furthermore, our results illustrate the utility of polyamine toxins as sensitive probes of AMPAR channel conductance and suggest the possibility that TARPs may influence their channel properties by selectively stabilizing specific channel conformations, rather than altering the pore structure.

\section{Introduction}

AMPARs are tetrameric assemblies of the subunits GluA1GluA4, and many aspects of their trafficking and gating depend on their specific subunit composition. The GluA2 subunit, in particular, exerts profound control over the functional properties

Received Dec. 21, 2010; revised March 2, 2011; accepted April 6, 2011.

Author contributions: A.C.J., A.D.M., D.S., M.F., S.G.C.-C., and R.A.N. designed research; A.C.J., A.D.M., and D.S. performed research; A.C.J., A.D.M., and D.S. analyzed data; A.C.., A.D.M., D.S., M.F., S.G.C.-C., and R.A.N. wrote the paper.

This work was supported by a Ruth L. Kirschstein National Research Service Award from the NIMH (F32MH081430 to A.C.J.), a graduate research fellowship from the National Science Foundation (to A.D.M.), and grants from the Wellcome Trust and the Royal Society (S.G.C.-C., M.F.). R.A.N. was supported by grants from the NIMH. We are indebted to A. Priel for invaluable help with oocyte recordings, helpful discussions, and critical reading of this manuscript. We thank D. Julius and members of the Julius lab for additional help with oocyte recordings, $M$. Hollmann for the generous gift of the pGEM-GluA4(Q) construct, A. Tzingounis and K. Bittner for helpful discussions and critical reading of this manuscript, K. Bjorgan and M. Cerpas for expert technical assistance, and members of the Cull-Candy/Farrant and Nicoll laboratories for helpful discussions.

*A.C.J., A.D.M., and D.S. contributed equally to this work.

**M.F., S.G.C.-C., and R.A.N. are co-senior authors.

Correspondence should be addressed to any of the following: Roger A. Nicoll, Department of Cellular and Molecular Pharmacology, University of California San Francisco, CA 94143, E-mail: nicoll@cmp.ucsf.edu; or Stuart G. Cull-Candy or Mark Farrant, Department of Neuroscience, Physiology, and Pharmacology, University College London, London WC1E 6BT, UK, E-mail: s.cull-candy@ucl.ac.uk or m.farrant@ucl.ac.uk.

A. D. Milstein's present address: Janelia Farm Research Campus, Howard Hughes Medical Institute, Ashburn, VA 20147.

D. Soto's present address: Laboratori de Neurobiologia, Institut d'Investigació Biomèdica de Bellvitge (IDIBELL), Feixa LLarga s/n, 08907 L'Hospitalet de Llobregat, Barcelona, Spain.

DOI:10.1523/JNEUROSCI.6688-10.2011

Copyright $\odot 2011$ the authors $\quad 0270-6474 / 11 / 317511-10 \$ 15.00 / 0$ of AMPARs (Malinow and Malenka, 2002; Bredt and Nicoll, 2003; Cull-Candy et al., 2006; Greger et al., 2007; Isaac et al., 2007; Ziff, 2007; Traynelis et al., 2010). GluA2 is subject to posttranscriptional RNA editing at the pore-lining Q/R site (Sommer et al., 1991), and incorporation of edited GluA2(R) subunits reduces the single-channel conductance of AMPARs (Swanson et al., 1997) and renders them impermeable to calcium (Hollmann et al., 1991; Burnashev et al., 1992). While GluA2-containing receptors exhibit a linear current-voltage relationship, AMPARs that lack GluA2 are subject to voltage-dependent block by endogenous intracellular polyamines such as spermine, resulting in strong inward rectification (Bowie and Mayer, 1995; Kamboj et al., 1995; Koh et al., 1995) and sensitivity to externally applied polyamine toxins (Blaschke et al., 1993; Herlitze et al., 1993; Washburn and Dingledine, 1996; Washburn et al., 1997; Toth and McBain, 1998), which block GluA2-lacking AMPARs in a noncompetitive use- and voltage-dependent manner (Strømgaard and Mellor, 2004; Strømgaard et al., 2005).

In addition to their pore-forming subunits, neuronal AMPARs are associated with auxiliary subunits-transmembrane AMPAR regulatory proteins (TARPs) — that act as powerful modulators of AMPAR channel properties. TARP association slows the kinetics of AMPAR activation, deactivation, and desensitization, and enhances agonist efficacy (Nicoll et al., 2006; Milstein and Nicoll, 2008; Coombs and Cull-Candy, 2009; Sager et 
al., 2009; Jackson and Nicoll, 2011b). The TARP family includes the prototypical TARP $\gamma-2$ (stargazin), as well as $\gamma-3, \gamma-4$, and $\gamma-8$ (Tomita et al., 2003). The homologous proteins $\gamma-5$ and $\gamma-7$ also share some functionality with TARPs (Kato et al., 2007, 2008; Soto et al., 2009). TARP association has been shown to enhance the mean channel conductance of homomeric, GluA2-lacking AMPARs (Soto et al., 2007; Suzuki et al., 2008; Soto et al., 2009), but diminish the affinity of the AMPAR pore for intracellular spermine (Soto et al., 2007). Furthermore, single-channel recordings showed that TARP $\gamma-2$ modulates GluA2-lacking AMPARs by increasing the duration of burst-like channel openings during prolonged agonist application (Tomita et al., 2005).

As the majority of neurons in the mammalian brain express the GluA2 subunit (Isaac et al., 2007) together with TARPs (Tomita et al., 2003, Menuz et al., 2007), we first sought to determine the channel properties of heteromeric, GluA2-containing AMPARs associated with different TARP subtypes. Although GluA2 incorporation reduces AMPAR channel conductance, we found that TARP association increases the mean channel conductance of GluA2-containing AMPARs in a TARPsubtype-specific manner. The AMPAR-TARP complexes that exhibited the greatest enhancement in mean channel conductance were also the ones more readily blocked by polyamine toxins. Furthermore, we found that AMPAR-TARP complexes activated by agonists of varying efficacy also exhibited differential block by polyamine toxins, consistent with the idea that polyamine toxins preferentially block AMPARs with higher mean channel conductance. Together, these data suggest that polyamine toxins are sensitive probes of AMPAR channel conductance, which varies with TARP subtype and agonist efficacy.

\section{Materials and Methods}

Human embryonic kidney $293 T$ cell recordings

Human embryonic kidney 293T (HEK293T; tsA201) cells were transfected with GluA2R(i), GluA4Q(i), $\gamma 2, \gamma-3, \gamma-4$, and $\gamma-8$ cDNAs in various combinations using Lipofectamine 2000 (Invitrogen) according to the manufacturer's instructions. The total amount of DNA used was 0.8 $\mathrm{mg}$, with the following ratios: GluA2:GluA4, 1:1; GluA2:GluA4: $\gamma$-x, $1: 1: 3$. Vectors containing cDNAs for $\gamma-2 \gamma-3, \gamma-4$, and $\gamma-8$ also contained cDNA encoding eGFP. For transfections lacking TARPs, $0.1 \mathrm{mg}$ eGFP cDNA was added.

Excised patch recordings. Outside-out membrane patches were excised from eGFP-expressing cells, and currents were recorded at room temperature $\left(22-24^{\circ} \mathrm{C}\right)$ with an Axopatch $200 \mathrm{~A}$ amplifier and acquired using a Digidata 1200 interface and pClamp software. The external solution contained (in mM) $145 \mathrm{NaCl}, 2.5 \mathrm{KCl}, 1 \mathrm{CaCl}_{2}, 1 \mathrm{MgCl}_{2}, 10$ glucose, and 10 HEPES, pH 7.3 with $\mathrm{NaOH}$. The intracellular (pipette) solution contained (in mM) $145 \mathrm{CsCl}, 2.5 \mathrm{NaCl}, 1 \mathrm{Cs}$-EGTA, $4 \mathrm{Mg}_{2} \mathrm{ATP}$, and 10 HEPES, pH 7.3 with CsOH. Spermine tetrahydrochloride (100 $\mu$ m; Tocris Bioscience) was added to the intracellular solution.

PhTx block experiments. Patches were placed in external solution containing cyclothiazide (CTZ; $50 \mu \mathrm{M}$; Ascent Scientific) and moved manually to a glutamate $(1 \mathrm{~mm})$ and CTZ-containing solution before being rapidly exposed to a solution with glutamate, CTZ, and philanthotoxin-74 (PhTx-74; $100 \mu \mathrm{M}$; Chiralix). The rapid switch to the PhTx74-containing solution was achieved using a theta-glass application tool moved by a piezoelectric device.

Patches were held at $-60 \mathrm{mV}$ during glutamate application (60 s). Two $+60 \mathrm{mV}$ pulses were applied during the record. The first, at the beginning of the record, was used to assess the degree of rectification. Records with a rectification index $(+60 \mathrm{mV} /-60 \mathrm{mV})$ below 0.8 were rejected. A second pulse, after the block, was used to relieve residual PhTx-74 block. Averaged currents describing onset of block were fitted with a double-exponential function:

$$
I=A_{\mathrm{f}} \exp \left(t / \tau_{\mathrm{f}}\right)+A_{\mathrm{s}} \exp \left(t / \tau_{\mathrm{s}}\right),
$$

where $A_{\mathrm{f}}$ and $\tau_{\mathrm{f}}$ are the amplitude and time constant of the fast component, and $A_{\mathrm{s}}$ and $\tau_{\mathrm{s}}$ are the amplitude and time constant of the slow component. The weighted time constant $\left(\tau_{\mathrm{w}}\right.$, block $)$ was calculated according to the following:

$$
\tau_{\mathrm{w}}=\tau_{\mathrm{f}}\left(\frac{A_{\mathrm{f}}}{A_{\mathrm{f}}+A_{\mathrm{s}}}\right)+\tau_{\mathrm{s}}\left(\frac{A_{\mathrm{s}}}{A_{\mathrm{f}}+A_{\mathrm{s}}}\right) .
$$

Fast agonist application to excised patches. Outside-out patches were obtained using electrodes fabricated from borosilicate glass (outer diameter, $1.5 \mathrm{~mm}$; inner diameter, $0.86 \mathrm{~mm}$; Harvard Apparatus) with a resistance of 8-12 $\mathrm{M} \Omega$. Rapid solution switching at the patch was achieved by piezoelectric translation of an application tool. The tool was made from theta glass (outer diameter, $2 \mathrm{~mm}$; Hilgenberg) pulled to a tip opening of $\sim 200 \mu \mathrm{m}$ and was mounted on a piezoelectric translator (Burleigh LSS-3000/PZ-150M, EXFO Life Sciences and Industrial Division; or P-265.00, Physik Instrumente). Control and agonist solutions flowed continuously through the two barrels, and solution exchange occurred when movement of the translator was triggered by a voltage step (pClamp). To enable visualization of the solution interface and allow measurement of solution exchange, $2.5 \mathrm{mg} / \mathrm{ml}$ sucrose was added to the agonist solution, and the control solution was diluted by $5 \%$. Recorded currents were low-pass filtered at $10 \mathrm{kHz}$ and digitized at 20 or 50 $\mathrm{kHz}$. At the end of each experiment, the adequacy of the solution exchange was assessed by destroying the patch and measuring liquidjunction current at the open pipette. Exchange 10-90\% rise times were typically between 100 and $300 \mu \mathrm{s}$. Nonstationary fluctuation analysis (NSFA) was used to determine the weighted mean single-channel conductance and the peak open probability $\left(P_{\mathrm{O}}\right.$, peak $)$, as described by Soto et al. (2007). Briefly, glutamate (10 mM) was applied to outside-out patches ( $100 \mathrm{~ms}$ duration; $1 \mathrm{~Hz}$ ), and the ensemble variance of all successive pairs of current responses was calculated using IGOR Pro 5.05 (Wavemetrics) and NeuroMatic (http://www.neuromatic.thinkrandom.com). The singlechannel current $(i)$ and total number of channels $(N)$ were then determined by plotting this ensemble variance $\left(\sigma^{2}\right)$ against mean current $(\bar{I})$ and fitting with a parabolic function:

$$
\sigma^{2}=i I-\bar{I}^{2} / N+\sigma_{\mathrm{B}}^{2},
$$

where $\sigma_{\mathrm{B}}{ }^{2}$ is the background variance. $P_{\mathrm{O} \text {, peak }}$ was calculated by dividing the average peak current $\left(I_{\text {peak }}\right)$ by $i N$. Steady-state open probability $\left(P_{\mathrm{O} \text {, steady-state }}\right)$ was calculated by multiplying $P_{\mathrm{O} \text {, peak }}$ by $I_{\text {steady-state }} / I_{\text {peak }}$, where $I_{\text {steady-state }}$ is the current remaining at the end of the $100 \mathrm{~ms}$ application. One caveat is that this approach to estimating $P_{\mathrm{O}}$, steady-state ignores the possibility that the unitary events contributing to the steadystate current have a different conductance than those contributing to the peak response.

\section{Cerebellar granule neuron recordings}

Preparation of stargazer cerebellar granule neuron cultures, transfection with TARP cDNA, and whole-cell recordings were performed as described previously (Milstein et al., 2007) using stargazer mutant mice of either sex. All experiments were carried out in accordance with animal welfare regulations set out by the University of California, San Francisco Institutional Animal Care and Use Committee. Agonist solutions were either $1 \mathrm{~mm}$ kainate or $1 \mathrm{~mm}$ glutamate with $100 \mu \mathrm{M}$ CTZ dissolved in external solution. Antagonists dissolved in agonist solution were either 100 or $300 \mu \mathrm{M}$ PhTx-74 (Chiralix). Solution exchange between control, agonist and agonist with antagonist was achieved using a local perfusion system (Automate Scientific).

\section{Oocyte recordings}

Two-electrode voltage-clamp (TEVC) recordings were performed on stage V-VI Xenopus laevis oocytes prepared as described previously (Ayalon and Stern-Bach, 2001; Priel et al., 2006). GluA4(Q)flip-pSGEM, generously provided by the laboratory of Dr. M. Hollmann (Ruhr University Bochum, Germany), was expressed in oocytes with and without TARPs $\gamma$ - 2 or $\gamma$ - 8 (in pGEM plasmid). GluA4 cRNA alone was injected at a concentration of $1 \mathrm{ng} / 50 \mathrm{nl}$. When coexpressed with TARPs, the cRNAs 
GluA2/4 Heteromers + TARPs in HEK293T Cells

A
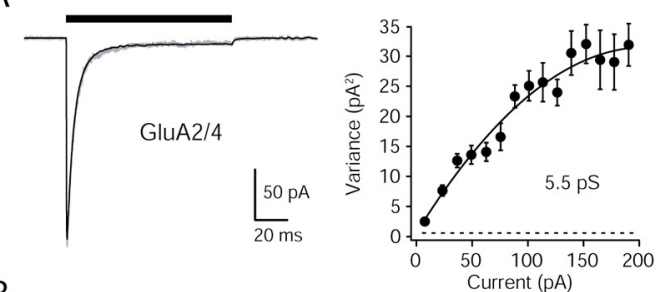

B
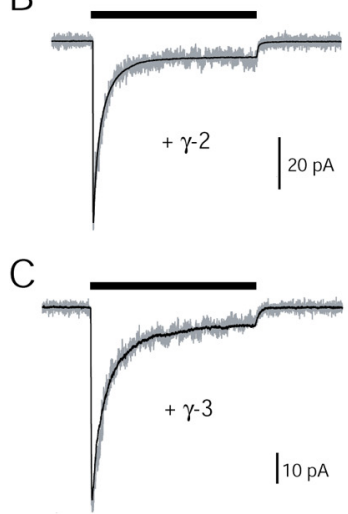

D
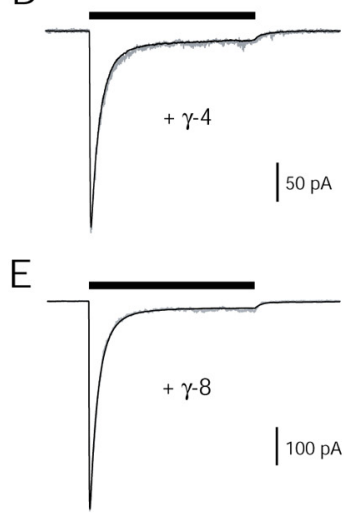
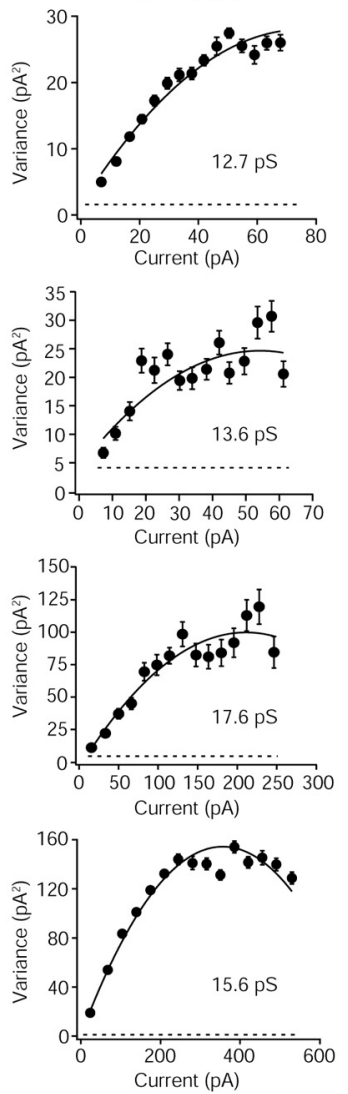

$\mathrm{F}$

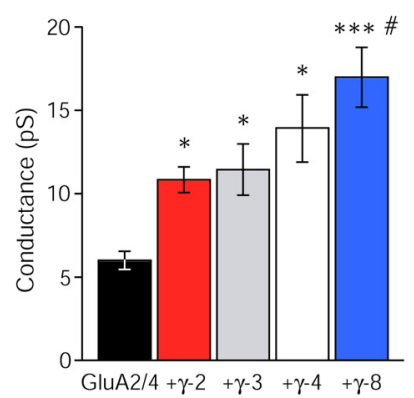

G

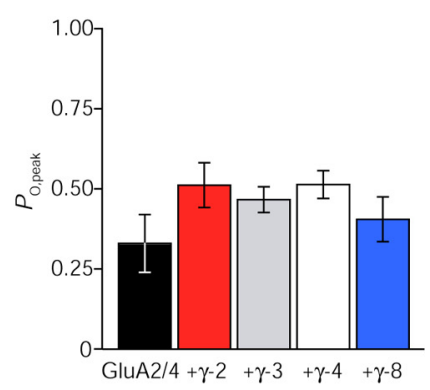

$\mathrm{H}$

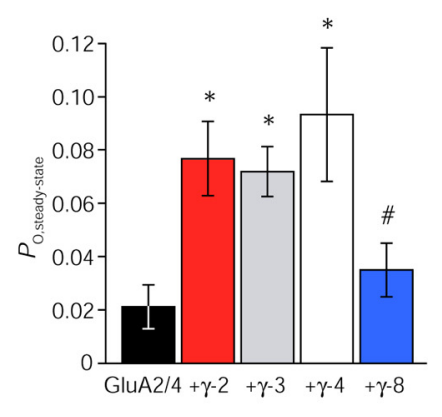

Figure 1. TARP subtypes differentially modulate channel properties of GluA2-containing AMPARs. $\boldsymbol{A}$, Left, Currents evoked by rapid application of $10 \mathrm{~mm}$ glutamate $(100 \mathrm{~ms},-60 \mathrm{mV})$ onto an outside-out patch from an HEK293T cell expressing heteromeric GluA2/A4 AMPARs. The black line is the mean of 54 traces; the gray line shows a representative single trace. Right, Current-variance relationship generated from NSFA of the recording shown on the left. For this patch, the fitted parabola gave an estimated weighted mean single-channel conductance of $5.5 \mathrm{pS}$. The dashed line denotes background variance. $\boldsymbol{B}-\boldsymbol{E}$, Currents evoked in the same conditions as in $\boldsymbol{A}$ from heteromeric GluA2/A4, each coexpressed with different TARP subtypes $(\gamma-2, \gamma-3, \gamma-4$, and $\gamma$-8) with corresponding current-variance relationships. $\boldsymbol{F}$, Bar graph comparing the effect of TARPs $\gamma-2, \gamma-3, \gamma-4$, and $\gamma-8$ on mean channel conductance of heteromeric GluA2/A4 AMPA receptors. Asterisks denote significance versus GluA2/A4 ( ${ }^{*} p<0.05$; ${ }^{* * *} p<0.0005$ ), and hash marks denotes significance relative to GluA2/A4 plus $\gamma-2\left({ }^{\#} p<0.05\right)$. G, Bar graph comparing the effect of TARPs $\gamma-2, \gamma-3, \gamma-4$, and $\gamma-8$ on $P_{0 \text {, peak }}$ of heteromeric GluA2/A4 AMPA receptors. $\boldsymbol{H}$, Bar graph comparing the effect of TARPs $\gamma-2, \gamma-3, \gamma-4$, and $\gamma-8$ on $P_{0 \text {, steady-state }}$ of heteromeric GluA2/A4 AMPARs. Error bars denote \pm SEM. Asterisks denote significance versus GluA2/A4 $\left({ }^{*} p<0.05\right)$, and hash marks denotes significance versus GluA2/A4 plus $\gamma-2\left({ }^{\#} p<0.05\right)$.

were expressed at the following ratio of GluA4 to TARP: 0.1:1 ng/50 nl. Oocyte recordings were performed 48 to $72 \mathrm{~h}$ after cRNA injection. TEVC recordings were performed at room temperature $\left(22-25^{\circ} \mathrm{C}\right)$ using a GeneClamp 500B amplifier connected to a Digidata 1322A digitizer and Clampex10.1 software (Molecular Devices). Borosilicate glass pipettes were pulled on a P-97 pipette puller (Sutter Instruments) to a resistance of $0.7-1 \mathrm{M} \Omega$ and filled with $3 \mathrm{M} \mathrm{KCl}$. A gravity-fed perfusion system and an oocyte perfusion chamber (Automate Scientific) were used for solution delivery and recording. Nominally $\mathrm{Ca}^{2+}$-free normal frog Ringer's solution [containing (in mM) $110 \mathrm{NaCl}, 2 \mathrm{KCl}, 1 \mathrm{MgSO}_{4}$, and 5 HEPES, pH 7.4] was used as the control solution. Agonists consisted of either $500 \mu \mathrm{M}$ kainate (Ascent Scientific) or $500 \mu \mathrm{M}$ glutamate (Sigma) with $100 \mu \mathrm{M}$ CTZ (Ascent
Scientific). Either 0.1 or $10 \mu \mathrm{M}$ philanthotoxin433 (PhTx-433; Chiralix) dissolved in agonist solution was then applied. All recordings were performed at a holding potential of $-70 \mathrm{mV}$.

\section{Data analysis and statistics}

Data were analyzed using the following software: Clampfit 10.1 (Molecular Devices), IgorPro 6.10 (WaveMetrics), MATLAB 7.10 (MathWorks), and Excel (Microsoft). Statistical significance was examined using a Wilcoxon rank sum test. Group differences were examined using a Kruskal-Wallis rank sum test followed by pairwise Wilcoxon rank sum tests with Holm's sequential Bonferroni correction (R 2.9.2, the R Project for Statistical Computing; http://www.R-project.org).

\section{Results \\ TARP subtypes differentially modulate channel properties of GluA2-containing AMPARs}

To examine the effects of TARPs on the channel properties of heteromeric, GluA2-containing AMPARs, we applied NSFA to currents evoked by ultrafast glutamate application onto outside-out patches from HEK293T cells coexpressing GluA2 and GluA4 (Fig. 1A). NSFA is a powerful method for estimating the single-channel properties underlying macroscopic currents (Sigworth, 1980; Robinson et al., 1991; Jonas et al., 1993; Traynelis et al., 1993; Benke et al., 1998) and is necessary for studying the exceptionally low-conductance single-channel currents arising from heteromeric GluA2containing AMPARs in the absence of TARPs (Swanson et al., 1997). GluA2/A4 heteromers were expressed with various TARP subtypes (Fig. $1 B-E$ ). Although no TARP subtype appreciably altered the peak open probability $\left(P_{\mathrm{O} \text {, peak }}\right)$ of GluA2/A4 heteromers $(p>0.1)$ (Fig. $1 G)$, TARP subtypes differentially modulated their steady-state open probability $\left(P_{\mathrm{O} \text {, steady-state }}\right)$ (see Materials and Methods) (Fig. $1 H$ ) and mean channel conductance (Fig. $1 F$ ). TARPs $\gamma-2, \gamma-3$, and $\gamma$-4 increased $P_{\mathrm{O}}$, steady-state approximately fourfold, whereas $\gamma$-8 had little effect (GluA2/A4, $n=9 ; \gamma-2, n=10, p<0.01 ; \gamma-3, n=12$, $p<0.005 ; \gamma-4, n=12, p<0.005 ; \gamma-8, n=$ $11, p<0.25)$. Interestingly, it was $\gamma-8$ that increased the mean channel conductance of GluA2/A4 heteromers most dramatically (approximately threefold), whereas $\gamma$-2, $\gamma-3$, and $\gamma-4$ were less effective (approximate twofold increase; $\gamma-2, p<0.005 ; \gamma-3, p<0.01 ; \gamma-4, p<0.005 ; \gamma-8, p<0.001$ relative to GluA2/A4, $p<0.05$ relative to $\gamma$-2). These data confirm that although GluA2 incorporation reduces the singlechannel conductance of AMPARs (Swanson et al., 1997), TARP association to some extent counteracts this influence by enhancing mean channel conductance and $P_{\mathrm{O}}$, steady-state, without significantly affecting $P_{\mathrm{O} \text {, peak }}$. 
TARP subtypes differentially modulate channel block of GluA2-containing AMPARs

One prediction of the increased mean channel conductance and $P_{\mathrm{O}}$, steady-state we observed for TARP-associated AMPARs is that the binding of molecules that interact directly with the channel pore will be facilitated. However, it has been demonstrated previously that TARPs decrease the sensitivity of AMPARs to block by intracellular polyamines such as spermine (Soto et al., 2007). If TARPs decrease AMPAR affinity for polyamines by disrupting their binding site in the channel pore, this would suggest that TARPs alter the structure of the AMPAR channel pore beyond simply modulating the relative stabilities and occupancies of preexisting channel conformations. Therefore, we sought to further probe the channel conformation of TARP-associated AMPARs by approaching the polyamine-binding site from the outside of the channel with polyamine philanthotoxins.

We again coexpressed AMPARs with and without TARPs in HEK293T cells and recorded currents from outside-out patches in response to ultrafast drug application. By rapidly stepping from an agonistcontaining solution to one also containing an antagonist, we were able to quantify the rate and extent of channel block (Fig. 2A$C)$. Whereas the classical channel blocker PhTx-433 is selective for GluA2-lacking, calcium-permeable AMPARs, a recently characterized variant, PhTx-74, blocks heteromeric GluA2-containing AMPARs at higher concentrations (Kromann et al., 2002; Nilsen and England, 2007), allowing investigation of the channel properties of this AMPAR, which is abundantly expressed in the brain yet historically less tractable through pharmacology. We observed that PhTx-74 blocks heteromeric, GluA2/A4 AMPARs in a voltage-dependent manner, qualitatively similar to that of PhTx433 on GluA2-lacking AMPARs (data not shown). We found that the rate (Fig. $2 A, B$ ) and extent (Fig. $2 A, C$ ) of block of GluA2/A4 heteromers by PhTx-74 was enhanced in the presence of TARPs, and that this effect varied among TARPs. Both the rate and extent of block were significantly greater for TARPs $\gamma$-4 and $\gamma-8$ than for $\gamma-2$ and $\gamma-3$.

To relate our findings from a heterologous system to neurons, we turned to a neuronal culture system that allows the expression and analysis of each TARP subtype in isolation. Cerebellar granule neurons (CGNs) in stargazer mice lack functional TARP $\gamma$ - 2 protein, causing a dramatic deficit in the trafficking of AMPARs to the cell surface. However, they natively express

B

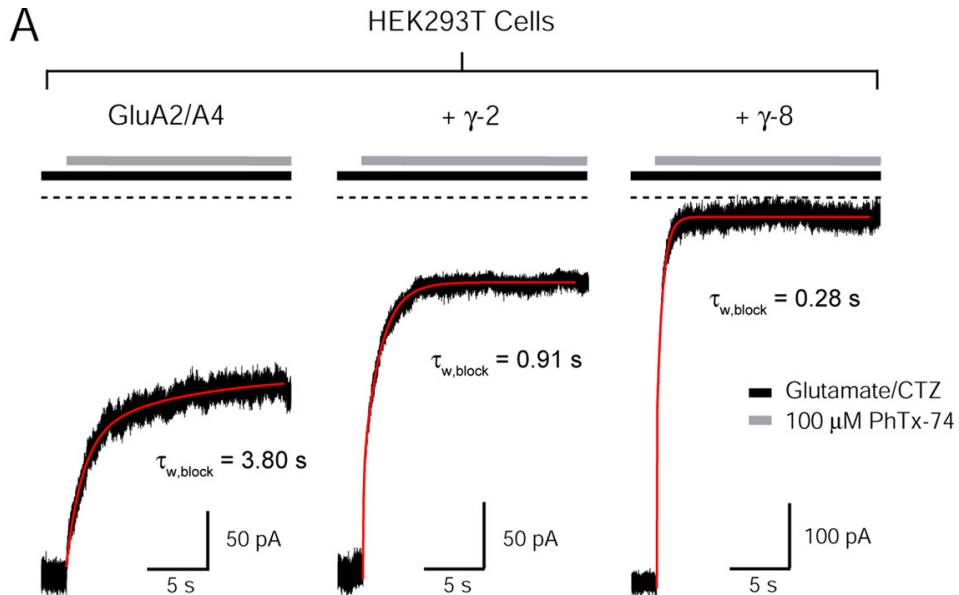

Rate of Block by $100 \mu \mathrm{M}$ PhTx-74

C

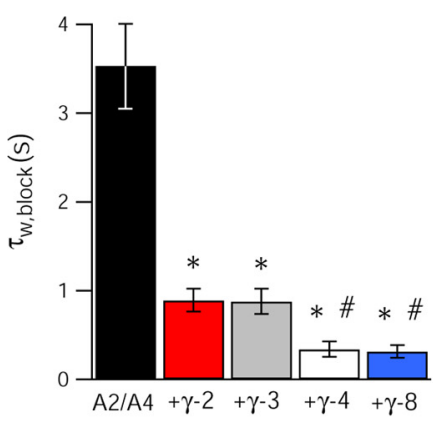

D

stg/stg Cerebellar Granule Neurons

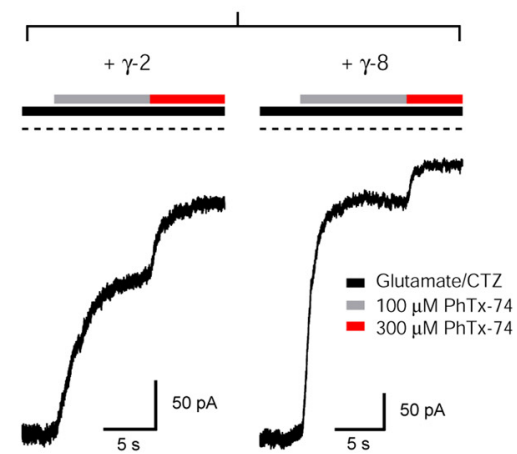

Extent of Block by $100 \mu \mathrm{M}$ PhTx-74

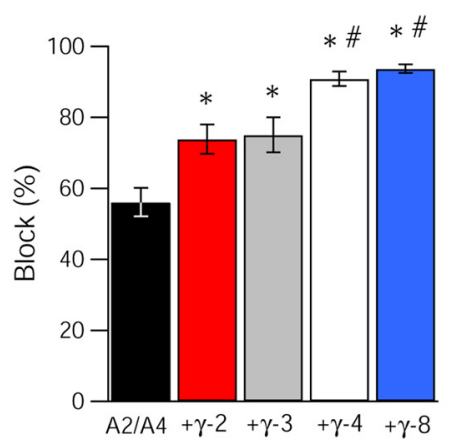

E
Extent of Block by $100 \mu \mathrm{M}$ PhTx-74

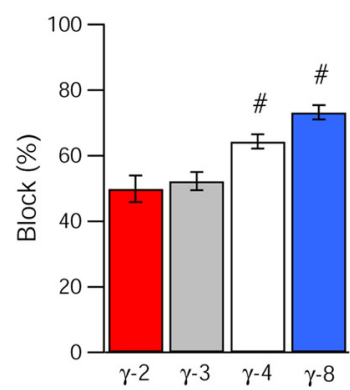

Figure 2. TARP subtypes differentially modulate channel block of GluA2-containing AMPARs. $A$, Representative traces demonstrating block by $100 \mu \mathrm{m} \mathrm{PhTx}-74$ of currents evoked by ultrafast application of $1 \mathrm{~mm}$ glutamate $+50 \mu \mathrm{m}$ CTZ, at a holding potential of $-60 \mathrm{mV}$, onto outside-out patches from HEK293T cells expressing GluA2/A4 AMPARs alone (left), GluA2/A4 with $\gamma-2$ (middle), and GluA2/4 with $\gamma-8$ (right). Onset of block was described by double-exponential functions (red lines) from which weighted time constants $\left(\tau_{w}\right.$, block $)$ were calculated. The rate and extent of block by PhTx-74 is increased in the presence of TARPs. B, Bar graph showing the rate of block $\left(\tau_{w, \text { block }}\right)$ of GluA2/A4 AMPARs by $100 \mu \mathrm{M} \mathrm{PhTX-74} \mathrm{in} \mathrm{HEK293T} \mathrm{cells} \mathrm{(mean} \pm$ SEM). Asterisks denote significance relative to GluA2/4 alone ( ${ }^{*} p<$ $0.05)$ and hash marks denote significance relative to $\gamma-2\left({ }^{\#} p<0.05\right)(n=7-9)$. C, Bar graph showing the extent of block of GluA2containing AMPARs by $100 \mu \mathrm{M} \mathrm{PhTx}-74$ in HEK293T cells (mean \pm SEM). Asterisks denote significance relative to GluA2/A4 alone ${ }^{*} p<$ $0.05)$ and hash marks denote significance relative to $\gamma-2\left({ }^{\#} p<0.05\right)(n=7-9) . D$, Representative traces demonstrating block by $100 \mu \mathrm{M}$ and $300 \mu \mathrm{mPhTx}$-74 of whole-cell currents evoked by $1 \mathrm{~mm}$ glutamate $+100 \mu \mathrm{m}$ CTZfrom GluA2-containing AMPARs in stg/stg cerebellar granule neurons transfected with TARPs $\gamma-2$ (left) and $\gamma-8$ (right). The extent of block of presumed GluA2/A4 heteromers is greater with TARP $\gamma-8$ than with $\gamma-2$. E, Bar graph showing the extent of block of GluA2-containing AMPARs by $100 \mu \mathrm{M} \mathrm{PhTx-74} \mathrm{in} \mathrm{stg/stg} \mathrm{granule}$ neurons (mean \pm SEM). Hash marks denote significance relative to $\gamma-2\left({ }^{\#} p<0.05\right)(n=9-10)$.

GluA2/A4 heteromeric AMPARs (Mosbacher et al., 1994; Swanson et al., 1997) that are retained intracellularly until their surface expression is "rescued" by exogenous TARP expression (Chen et al., 2000; Milstein et al., 2007). We made whole-cell recordings 


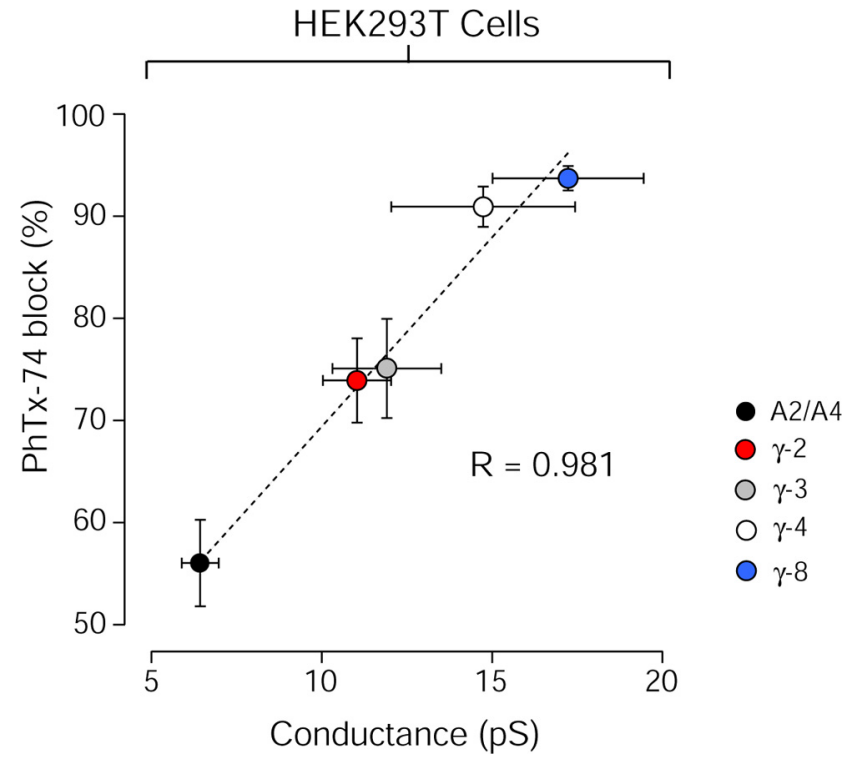

Figure 3. Linear correlation between mean channel conductance and channel block of GluA2-containing AMPARs. The relationship between mean channel conductance (from Fig. $1 \mathrm{~F}$ ) and percentage block by $100 \mu \mathrm{m} \mathrm{PhTx}-74$ (from Fig. 2O) for GluA2/A4 heteromeric AMPARs coexpressed with TARPs $\gamma-2, \gamma-3, \gamma-4$, and $\gamma-8$ in HEK293T cells is shown. The dashed line shows the weighted total least squares fit ( $R$ is the weighted correlation coefficient). Symbols and error bars denote mean \pm SEM.

from stargazer CGNs expressing various TARP subtypes and evoked AMPAR-mediated currents by locally applying agonist to the cell body. We then measured the use-dependent block of these currents by PhTx-74. The TARP subtype specificity of channel block we had observed in HEK293T cells was recapitulated in these neurons. Thus, AMPARs associated with $\gamma-4$ and $\gamma$-8 displayed more potent block than those containing $\gamma-2$ or $\gamma-3$ (Fig. $2 D, E$ ). In the same experiments, we confirmed that surfaceexpressed AMPARs were GluA2 containing by demonstrating that the extent of block by $10 \mu \mathrm{M}$ PhTx-433 was negligible (data not shown). Interestingly, in both systems it was AMPARs coexpressed with $\gamma-8$ that exhibited the highest sensitivity to PhTx-74 (Fig. 2C,E), despite having the lowest probability of opening (Fig. $1 \mathrm{H})$. However, $\gamma-8$ increased the mean channel conductance of AMPARs to the greatest extent, suggesting that this feature may be the more important in determining channel block by polyamines. Although the pattern of TARP-subtype-dependent block was the same between HEK293T cells and staragazer CGNs, the extent of block was found to be generally higher in the former than the latter. This difference may be attributed to the method of drug application or differential TARP stoichiometry (Shi et al., 2009; Kim et al., 2010). Nevertheless, our data revealed a linear correlation between the extent of channel block by PhTx-74 and the mean channel conductance of GluA2-containing AMPARs expressed in HEK293T cells (Fig. 3). These data demonstrate that the polyamine-binding site, accessible from the extracellular side of the membrane, remains intact when AMPARs are associated with TARPs. Furthermore, our data raise the possibility that polyamines may preferentially bind to open AMPAR channels in their highest subconductance state, as these have been shown to predominate when AMPARs are associated with TARP $\gamma$-2 (Tomita et al. 2005). Alternate possibilities are considered below (see Discussion), in addition to a model to reconcile these data with the previously described TARP-dependent reduction in the sensitivity of the AMPAR pore to intracellular spermine (Soto et al., 2007).

\section{Channel block of GluA2-containing AMPARs depends on agonist efficacy}

Thus far, our results suggest that TARP subtypes differentially modulate AMPAR mean channel conductance in a way that influences the rate and extent of channel block by PhTx-74. PhTx-74 appears to most effectively block channels associated with TARP subtypes conferring the highest mean channel conductance. One possible explanation for this behavior is that different TARPs confer specific AMPAR channel conformations that can be inferred from the differential sensitivity to PhTx block. Another possibility is that TARPs act to bias the relative distribution of preexisting single-channel subconductance states that are intrinsic to the AMPAR channel itself. If the latter were true, we might expect to observe TARP-dependent and -independent differences in PhTx efficacy using different AMPAR agonists, which confer differing single-channel properties (Cull-Candy and Usowicz, 1987; Jahr and Stevens, 1987; Ascher and Nowak, 1988). For this purpose, we examined the differential effects of full and partial agonists on GluA2-containing AMPARs. Previous work has shown that AMPARs activated by partial agonists such as kainate exhibit smaller amplitude macroscopic currents and preferentially occupy lower conductance channel states than those activated by full agonists such as glutamate (Swanson et al., 1997; Jin et al., 2003).

We first compared the agonist dependence of PhTx-74 block of GluA2/A4 heteromers in HEK293T cells, coexpressed with different TARPs, again using ultrafast drug application. We chose to focus on the two TARP subtypes that exhibited the most divergent values of mean channel conductance and extent of block: $\gamma-2$ and $\gamma-8$. We found that TARP-associated GluA2/A4 heteromers activated by kainate exhibited the same pattern of TARPsubtype-dependent block as those activated by glutamate/CTZ: heteromers associated with $\gamma-8$ were blocked significantly more effectively by PhTx-74 than those associated with $\gamma$-2, which in turn were blocked more effectively than heteromers alone (Fig. $4 A, B)$. But in each condition, PhTx-74 efficacy was higher when using glutamate/CTZ as an agonist compared with kainate (Fig. $4 B$ ). Interestingly, we observed that the efficacy of $\mathrm{PhTx}-74$ block of kainate-evoked current from $\gamma$-2-associated heteromers matched that of glutamate-evoked current from heteromers alone, whereas the efficacy of block of kainate-evoked current from $\gamma$-8-associated heteromers matched that of glutamateevoked current from $\gamma$-2-associated heteromers (Fig. $4 B$ ). Together, these data suggest that PhTx more effectively blocks AMPARs activated by a full agonist than a partial agonist, consistent with the notion that the toxin preferentially blocks AMPARs that exhibit a higher conductance.

\section{Channel block of GluA2-lacking AMPARs depends on TARP association and agonist efficacy}

The differential agonist effects we observed with GluA2-containing AMPARs led us to examine the possibility that channel block of GluA2-lacking AMPARs is subject to similar agonist- and TARPdependent modulation. In addition, since GluA2-containing AMPARs are not subject to block by intracellular polyamines, GluA2-lacking AMPARs would provide a way of more directly comparing the role of TARPs in modulating polyamine binding from both the intracellular and extracellular faces of the AMPAR pore. Previous work on Xenopus oocytes showed that $10 \mu \mathrm{M}$ PhTx-433 blocks currents through GluA3 homomeric AMPARs, while leaving GluA2/GluA3 heteromeric AMPARs unaffected (Washburn and Dingledine, 1996). Similar results were obtained for block of GluA1 homomeric AMPARs with the related compound PhTx-343 
(Brackley et al., 1993). In these studies, however, AMPARs were activated with the partial agonist kainate, in the absence of TARPs. To determine whether agonist efficacy affects the ability of PhTx-433 to block GluA2-lacking AMPAR channels, we compared the extent of block for currents evoked either by kainate or glutamate.

We first expressed either GluA3 or GluA4 in Xenopus oocytes and measured the percentage block of kainate-evoked currents by either 0.1 or $10 \mu \mathrm{M}$ PhTx-433, concentrations that encompassed the region of greatest slope in the dose-response curve for PhTx-433 (Washburn and Dingledine, 1996). With either GluA4 (Fig. $5 A, C$ ) or GluA3 (data not shown), the low concentration of PhTx-433 (0.1 $\mu \mathrm{M})$ had little effect on kainate-evoked currents, slowly blocking $14.5 \pm 1.7 \%(n=19)$ of the response. The high concentration (10 $\mu \mathrm{M})$ produced a rapid and near-complete block (93.6 $\pm 0.9 \% ; n=19$ ) (Fig. $5 A, C$ ), confirming previous observations (Washburn and Dingledine, 1996). We next used the same two concentrations of PhTx-433 on currents evoked by the full agonist, glutamate, the rapid desensitization of which was prevented by the addition of CTZ (Partin et al., 1995). Both 0.1 and $10 \mu \mathrm{M}$ PhTx-433 produced near-complete block of glutamate/CTZ-evoked currents $(0.1 \mu \mathrm{M}$, $90.4 \pm 1.2 \%, n=14 ; 10 \mu \mathrm{M}, 99.1 \pm 0.2 \%$, $n=13$ ) (Fig. $5 B, C$ ). These data suggest that PhTx-433 block of GluA2-lacking AMPAR channels is even more steeply agonist dependent than PhTx-74 block of GluA2-containing receptors. AMPAR channels activated by the full agonist glutamate, which gives rise to higher conductance openings, are more effectively blocked by PhTx than those activated by the partial agonist kainate.

Given that TARP association dramatically enhances the kainate efficacy at AMPARs (Tomita et al., 2005; Turetsky et al., 2005; Milstein and Nicoll, 2008), we hypothesized that PhTx-433 block of kainate-evoked AMPAR currents would be enhanced by TARPs. We coexpressed GluA4 with either TARP $\gamma-2$ or $\gamma-8$ (again using the two TARP subtypes that exhibited the most divergent mean channel conductance values) in Xenopus oocytes and confirmed that TARP association significantly enhanced kainate efficacy (data not shown). Whereas kainate responses from GluA4 homomers alone were blocked by $\sim 15 \%$ by $0.1 \mu \mathrm{M}$ PhTx433, responses from GluA4 homomers coexpressed with either TARP $\gamma$-2 or $\gamma-8$ were subject to near-complete block $(\gamma-2$, $90.2 \pm 1.0 \%, n=23 ; \gamma-8,94 \pm 0.6 \%, n=23$ ) (Fig. $5 A, C$ ). Unlike the results obtained using PhTx-74 to block GluA2-containing receptors with $0.1 \mu \mathrm{M} P \mathrm{PhT}_{\mathrm{T}} 433$, we observed an agonistdependent difference in PhTx efficacy only for TARP-less GluA4 homomers. The efficacy of PhTx-433 block of kainate-evoked currents from both $\gamma$-2- and $\gamma$-8-associated GluA4 homomers matched that of glutamate-evoked current from the homomers alone (Fig. $5 B, C$ ). Just as the low concentration of PhTx-433 (0.1 $\mu \mathrm{M})$ produced nearly complete block in both cases, and did not reveal a differential effect of TARPs, an even lower concentration
$(0.01 \mu \mathrm{M})$ did not exhibit differential block of kainate-evoked currents $(\gamma-2,62.7 \pm 4.8 \%, n=4 ; \gamma-8,44.7 \pm 7.9 \%, n=7 ; p=$ 0.79 , Wilcoxon rank sum test). It is interesting to note that a recent study described a TARP-dependent difference in the efficacy of NASPM block of GluA1 homomers (Kott et al., 2009), specifically a lack of effect of TARP $\gamma$-4. Nevertheless, our data support the notion that the higher conductance openings activated by a full agonist are likely to be more effectively blocked by PhTx-433 than those activated by a partial agonist.

Furthermore, given the ability of CTZ to enhance AMPAR mean channel conductance and open probability (Fucile et al., 2006), we asked whether CTZ alone might occlude the agonistand TARP-dependent enhancement in PhTx-433 block shown in Figure 5. Although a saturating concentration of CTZ $(100 \mu \mathrm{M})$ enhanced PhTx-433 (0.1 $\mu \mathrm{M})$ block of KA-evoked current on its own, it could not account for the dramatic agonist-dependent enhancement in block (38.6 $\pm 6.3 \%, n=8 ; p<0.0005$ relative to KA alone; $p<0.0001$ relative to glutamate/CTZ) (data not shown). Also, in the presence of $\gamma-2$, CTZ did not confer any additional enhancement in block of KA-evoked currents (89.6 \pm $1.7 \%, n=7 ; p<0.0005$ relative to KA/CTZ in the absence of $\gamma$-2; $p=0.7$ relative to kainate alone in the presence of $\gamma-2$ ) (data not shown), indicating that CTZ does not occlude TARP-dependent effects on block. These results are also relevant to Figure 2, where the use of CTZ was necessary to assess PhTx-74 block of GluA2/ A4 heteromers. 


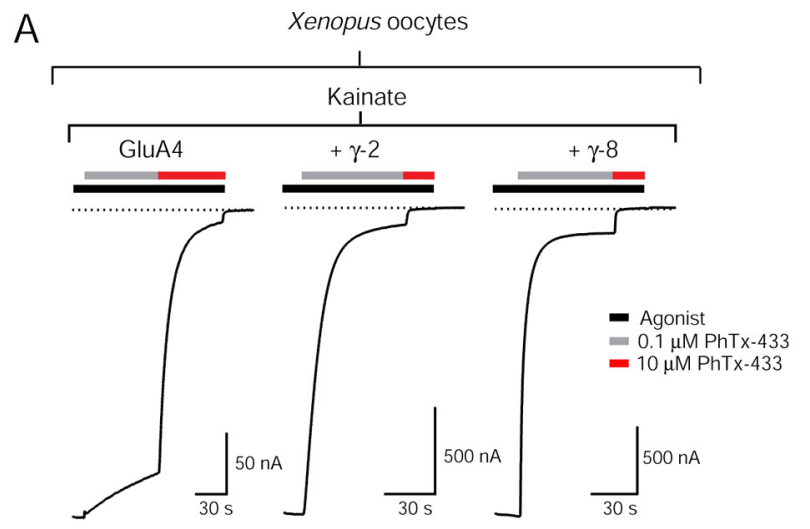

B

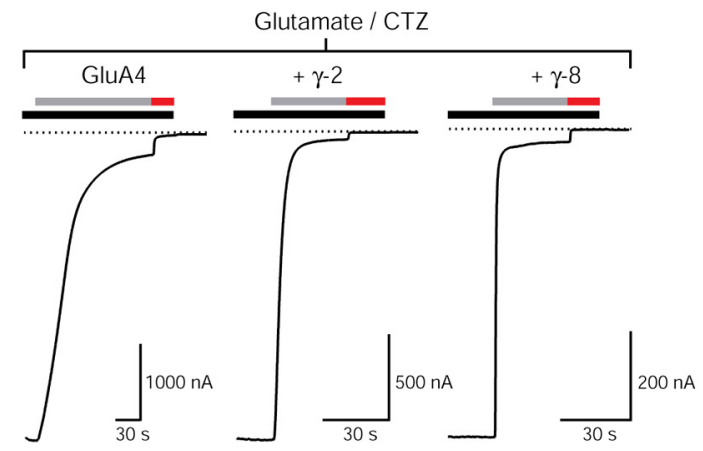

C

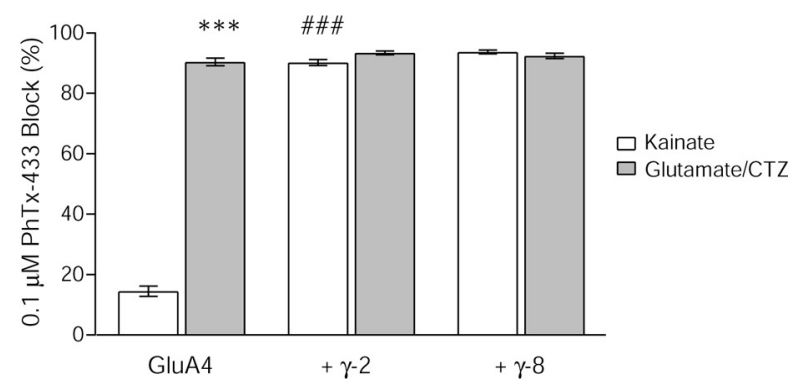

Figure 5. Channel block of GluA2-lacking AMPARs by PhTx-433 depends on agonist efficacy and TARP association. Representative traces of TEVC recordings from Xenopus oocytes showing the agonist and TARP dependence of block by PhTx-433. A, Block of kainate-evoked (500 $\mu \mathrm{m}$ ) AMPAR currents by two concentrations of PhTx-433. The low concentration of PhTx-433 (0.1 $\mu \mathrm{M})$ was relatively ineffective at blocking current through GluA4 homomers, whereas a high concentration $(10 \mu \mathrm{m})$ produced near-complete block. Coexpression with either TARP $\gamma$-2 or $\gamma$-8 greatly enhanced block by $0.1 \mu \mathrm{M}$ PhTx-433. B, Block of glutamate-evoked (500 $\mu \mathrm{m}$ ) and cyclothiazide-evoked (100 $\mu \mathrm{m})$ AMPAR currents evoked by two concentrations of PhTx-433. The low concentration of PhTx-433 $(0.1 \mu \mathrm{m})$ produced near-complete block of current through GluA4 homomers. Coexpression with either TARP $\gamma-2$ or $\gamma-8$ also produced near-complete block by $0.1 \mu \mathrm{m}$ PhTx-433. C, Bar graph showing the extent of block of GluA2-lacking AMPARs with and without TARPs by the low concentration of PhTx-433 $(0.1 \mu \mathrm{m})$ in Xenopus 0ocytes (mean \pm SEM). Block of currents evoked by kainate (open bars) and glutamate/CTZ (filled bars) is shown for each condition. Asterisks denote the significance between kainate and glutamate/ CTZ conditions ( ${ }^{* *} p<0.0001$, significant difference for GluA4 homomers alone; $n=14-19$ ). Hash marks denote the significance between kainate-evoked currents from TARP-associated GluA4 homomers relative to homomers alone ( ${ }^{\# \# \#} p<0.0001$, significant difference in block relative to homomers alone; $n=19-23$ ).

\section{Agonist dependence of channel block of GluA2-lacking AMPARs in neurons}

Finally, we sought to relate these findings to AMPARs in neurons and again turned to CGNs. This time, we used CGNs cultured from GluA2 knock-out (GluA2 ${ }^{-/-}$) mice, reasoning that these neurons would primarily express GluA4 homomeric AMPARs associated with TARP $\gamma$-2 and would represent a pure population of $\gamma$-2 associated GluA2-lacking receptors that could be directly compared with recombinant GluA4 coexpressed with $\gamma-2$ in oocytes. If PhTx-433 behaves in the same manner on neuronal AMPARs as it does on those in oocytes, kainate-evoked currents should be effectively blocked by the low concentration $(0.1 \mu \mathrm{M})$ of the toxin. We found that both kainate- and glutamate/CTZevoked currents in GluA2 ${ }^{-1-}$ CGNs were indeed strongly blocked by $0.1 \mu \mathrm{M}$ PhTx-433 $(84.5 \pm 3.7 \% ; n=10)($ Fig. $6 A, B)$. These data demonstrate that TARP-associated AMPARs occupy channel states with a high sensitivity to $\mathrm{PhTx}$-433. Interestingly, these same TARP-associated GluA2-lacking AMPARs exhibit a reduced sensitivity to block by intracellular spermine (Soto et al., 2007) (see Discussion).

\section{Discussion}

Our experiments have revealed three main findings. First, TARP auxiliary subunits differentially modulate the open probability and mean channel conductance of GluA2-containing AMPARs. Second, TARP subtypes also differentially modulate the rate and extent of channel block of GluA2-containing AMPARs by PhTx74. They do this in a manner that correlates with their effects on mean channel conductance. Third, PhTx block of both GluA2containing and -lacking AMPAR channels depends on agonist efficacy, being considerably more potent for the full agonist glutamate than the partial agonist kainate.

Based on these results, we can infer a certain amount of structural information about how TARPs modulate AMPAR channel conformation. The model that emerges from these data is that, despite the constraining effect of the GluA2 subunit, TARPs are able to tune the mean channel conductance of GluA2-containing AMPARs in a subtype-specific manner. Functional and structural data suggest that single AMPARs can open to various discrete multiple conductance levels (Cull-Candy and Usowicz, 1987; Jahr and Stevens, 1987; Ascher and Nowak, 1988). These display agonist concentration dependence (Rosenmund et al. 1998; Smith and Howe, 2000; Gebhardt and Cull-Candy, 2006; Prieto and Wollmuth, 2010), which likely corresponds to distinct conformational states adopted by the ligand-binding domains (Jin et al., 2003). Furthermore, the relative stabilities of various closed-channel states influence key macroscopic properties, including the amplitude and kinetics, of synaptic currents (Zhang et al., 2006, 2008). Our data are reasonably consistent with the view that TARP association enhances the mean channel conductance of AMPARs through a shift in the distribution of discrete subconductance states, favoring higher conductance openings (Tomita et al., 2005). In this case, increasing the occupancy of the highest conductance channel states may occur in concert with decreased occupancy of lower conductance channel states (or increased occupancy of various closed-channel states) such that the overall $P_{\mathrm{O}}$ remains unchanged (Fig. 1). Our finding that channel conductance, but not peak or steady-state $P_{\mathrm{O}}$, correlates with PhTx block, suggests that the interaction of polyaminebased toxins with the AMPAR pore is sensitive to the variety of conformational states that manifest as changes in mean channel conductance.

An open question with regard to our data is the degree to which the mechanism underlying TARP-subtype-dependent modulation of mean channel conductance is indeed a function of discrete structural differences between TARP subtypes. Sequence alignment shows that the canonical TARP family members $(\gamma-2$, $\gamma-3, \gamma-4$, and $\gamma-8)$ are highly homologous to each other although $\gamma-2 / \gamma-3$ and $\gamma-4 / \gamma-8$ are especially so (Klugbauer et al., 2000; Burgess et al., 2001; Sager et al., 2009). Furthermore, $\gamma-8$ is 
unique among the TARPs in having a particularly long C terminal (Burgess et al., 2001). Despite this high degree of homology, specific protein domains can account for TARP-subtype-specific effects on gating (Cho et al., 2007; Milstein et al., 2007; Milstein and Nicoll, 2009). This suggests that there may indeed be structural elements, unique to specific TARP subtypes, which determine AMPAR gating properties such as single-channel conductance. Another possibility is that the differential effects on mean channel conductance reflect TARP-subtype-specific differences in the stoichiometry of AMPAR-TARP complexes, which have been shown to vary in different preparations (Shi et al., 2009; Kim et al., 2010). Nevertheless, our data showing that TARP subtypes differentially modulate the channel properties of GluA2-containing AMPARs reinforces the view that the peak current and kinetics of synaptic AMPARs in the CNS will vary across neuronal cell-types, depending on the specific TARP subtypes expressed, their levels of expression, and AMPAR-TARP stoichiometry.

Recent work showed that TARP association reduces the sensitivity of GluA2-lacking AMPARs to block by the endogenous intracellular polyamines, spermine, and spermidine (Soto et al., 2007). These data raised the possibility that TARPs modulate AMPAR function not only by selectively stabilizing specific AMPAR channel conformations, but also by fundamentally altering the structure of the AMPAR pore such that the binding site for spermine is disrupted (Milstein and Nicoll, 2008). A priori, this "altered pore" model of TARP modulation might be taken to predict that AMPAR channel block by externally applied polyamine toxins would also be reduced in the presence of TARPs, if the molecules share the same site of interaction in the AMPAR channel pore (Andersen et al., 2006; Tikhonov, 2007). However, we observed a TARP-dependent increase in channel block by polyamine toxins of both GluA2-containing and GluA2-lacking AMPARs, counter to that observed with intracellular spermine. Thus, our data appear inconsistent with a model in which TARPs simply disrupt the polyamine-binding sites.

The fact that TARP association has seemingly opposing effects on AMPAR block might appear as something of a paradox. However, the idea that higher conductance channels are more sensitive to block by extracellular PhTx, but less sensitive to intracellular spermine, is not necessarily contradictory and has several possible interpretations. It may reflect the relative abilities of these two molecules to permeate the AMPAR pore. Previous work showed that intracellular spermine blocks glutamate receptor channels at moderately depolarized membrane potentials, but as the driving force increases, the positively charged spermine permeates the channel, resulting in a doubly rectifying $I-V$ with outward rectification in the more positive voltage range (Bowie and Mayer, 1995; Bähring et al., 1997; Bähring and Mayer, 1998; Bowie et al., 1998; Panchenko et al., 1999). PhTx resembles spermine in being weakly permeant, with strong hyperpolarization causing dissociation and permeation of toxin, although in this case dissociation triggers entry of the channel into a closed blocked state (Bähring et al. 1998). Molecular modeling suggested that the uncharged head group of PhTx interacts with, and is anchored by, the Q/R site at the narrowest constriction of the pore, whereas the charged polyamine tail extends deeper within the pore and interacts spe- cifically with glycine residues that line the pore (Andersen et al., 2006; Tikhonov, 2007). It is possible that those residues involved in binding of PhTx from the extracellular side are more readily accessible when AMPARs open to their highest subconductance states, a feature that is associated with the presence of TARPs. For spermine, however, the conformational state associated with large conductance openings might tend to facilitate its outward permeation through the AMPAR channel pore. Previous work (Bowie et al., 1998) has shown that intracellular block by spermine is reduced when AMPAR channel conductance is experimentally increased by altering the ionic driving force. Thus, it could be expected that block will occur more readily when ion flux (channel conductance) is low. However, our data indicate that polyamine block is also sensitive to the conformational state of the AMPAR channel such that externally applied PhTx has increased access to its binding site(s) in the AMPAR pore when the channel is in the conformations corresponding to its largest subconductance states. Although consistent with a straightforward effect on open-channel block, our data do not exclude the possibility that the effects of TARPs on equilibrium block reflect, in part, altered trapping or permeation of $\mathrm{PhTx}$.

The agonist-dependent effects we observed are likely explained by the fact that AMPARs activated by kainate exhibit significantly lower mean single-channel conductances than those activated by glutamate. Previous single-channel recordings demonstrated that GluA4 homomeric AMPARs activated by glutamate exhibit an array of distinct subconductance states (Swanson et al., 1997; Tomita et al., 2005). However, single-channel recordings of AMPARs activated by kainate revealed channel openings that were too small, brief, and infrequent to permit detailed analysis (Swanson et al., 1997). As a proxy, spectral analysis of current noise was used to estimate the single-channel conductance of recombinant GluA4 homomeric receptors activated by kainate to be 2.5 pS (Swanson et al., 1997). Furthermore, single-channel recordings from unedited nondesensitizing GluA2 homomers using 5-substituted willardiines revealed that although these partial agonists activate essentially the same subconductance states as a full agonist, they preferentially activate the lower levels (Jin et al., 2003). Furthermore, our data showing that PhTx-433 block of GluA2-lacking AMPARs is profoundly agonist dependent resolve an apparent discrepancy between earlier studies reporting very different affinities of PhTx-343 for homomeric AMPARs. Whereas Brackley et al. (1993) had reported that PhTx-343 blocked GluA1 homomers with an $\mathrm{IC}_{50}$ of $\sim 3 \mu \mathrm{M}$ when currents were evoked by kainate, Bähring and Mayer (1998) described 
preliminary experiments where glutamate/CTZ-evoked currents through GluA1 homomers were almost completely blocked by $0.03 \mu \mathrm{M}$ PhTx-343. Moreover, recent work showed that glutamate/CTZ-evoked currents from GluA2-lacking AMPARs in hippocampal and cerebellar neurons could be completely blocked by $0.1 \mu \mathrm{M}$ PhTx-433 (Lu et al., 2007; Jackson and Nicoll, 2011a). Our results confirm that PhTx blocks AMPARs with a dramatically higher potency when glutamate is used as an agonist when compared to kainate. Although in principle the reduced efficacy of kainate could reflect lower channel open probability, the accepted view is that it preferentially opens channels to low conductance states. This makes it tempting to suggest that it is this feature that underlies the differential blocking action of $\mathrm{PhTx}$ (Jin et al. 2003).

What significance do our findings have for the interpretation of PhTx block of AMPARs in neurons? As the majority of neuronal AMPARs are associated with TARPs, our results indicate that native AMPARs are likely to be dramatically more sensitive to PhTx block than previously expected from experiments on recombinant receptors. Additionally, measurements of PhTx block will reflect not only the subunit composition of AMPARs, but also their mean conductance, which can be modulated by TARP auxiliary subunits, and potentially by posttranslational modification of the AMPAR subunits themselves (Derkach et al., 1999). Furthermore, our observations suggest that the AMPAR channel pore region may be able to adopt an array of agonist- and TARPsubtype-dependent conformational states that can be probed using commonly used AMPAR channel blockers. This suggests that these compounds would provide sensitive probes of changes in AMPAR conductance such as those which occur as a result of synaptic plasticity (Benke et al., 1998). Finally, in light of the recent crystallization of the AMPAR (Sobolevsky et al., 2009), such tools could be invaluable in furthering our understanding of the fine structure of the AMPAR pore region.

\section{References}

Andersen TF, Tikhonov DB, Bolcho U, Bolshakov K, Nelson JK, Pluteanu F, Mellor IR, Egebjerg J, Stromgaard K (2006) Uncompetitive antagonism of AMPA receptors: mechanistic insights from studies of polyamine toxin derivatives. J Med Chem 49:5414-5423.

Ascher P, Nowak L (1988) Quisqualate- and kainate-activated channels in mouse central neurones in culture. J Physiol 399:227-245.

Ayalon G, Stern-Bach Y (2001) Functional assembly of AMPA and kainate receptors is mediated by several discrete protein-protein interactions. Neuron 31:103-113.

Bähring R, Mayer ML (1998) An analysis of philanthotoxin block for recombinant rat GluR6(Q) glutamate receptor channels. J Physiol 509:635-650.

Bähring R, Bowie D, Benveniste M, Mayer ML (1997) Permeation and block of rat GluR6 glutamate receptor channels by internal and external polyamines. J Physiol 502:575-589.

Benke TA, Luthi A, Isaac JT, Collingridge GL (1998) Modulation of AMPA receptor unitary conductance by synaptic activity. Nature 393:793-797.

Blaschke M, Keller BU, Rivosecchi R, Hollmann M, Heinemann S, Konnerth A (1993) A single amino acid determines the subunit-specific spider toxin block of alpha-amino-3-hydroxy-5-methylisoxazole-4-propionate/ kainate receptor channels. Proc Natl Acad Sci U S A 90:6528-6532.

Bowie D, Mayer ML (1995) Inward rectification of both AMPA and kainate subtype glutamate receptors generated by polyamine-mediated ion channel block. Neuron 15:453-462.

Bowie D, Lange GD, Mayer ML (1998) Activity-dependent modulation of glutamate receptors by polyamines. J Neurosci 18:8175-8185.

Brackley PT, Bell DR, Choi SK, Nakanishi K, Usherwood PN (1993) Selective antagonism of native and cloned kainate and NMDA receptors by polyamine-containing toxins. J Pharmacol Exp Ther 266:1573-1580.

Bredt DS, Nicoll RA (2003) AMPA receptor trafficking at excitatory synapses. Neuron 40:361-379.
Burgess DL, Gefrides LA, Poreman PJ, Noebels JL (2001) A cluster of three novel Ca2 + channel $\gamma$ subunit genes on chromosome 19q13.4: evolution and expression profile of the $\gamma$ subunit gene family. Genomics 71:339-350.

Burnashev N, Schoepfer R, Monyer H, Ruppersberg JP, Gunther W, Seeburg PH, Sakmann B (1992) Control by asparagine residues of calcium permeability and magnesium blockade in the NMDA receptor. Science 257:1415-1419.

Chen L, Chetkovich DM, Petralia RS, Sweeney NT, Kawasaki Y, Wenthold RJ, Bredt DS, Nicoll RA (2000) Stargazin regulates synaptic targeting of AMPA receptors by two distinct mechanisms. Nature 408:936-943.

Cho CH, St-Gelais F, Zhang W, Tomita S, Howe JR (2007) Two families of TARP isoforms that have distinct effects on the kinetic properties of AMPA receptors and synaptic currents. Neuron 55:890-904.

Coombs ID, Cull-Candy SG (2009) Transmembrane AMPA receptor regulatory proteins and AMPA receptor function in the cerebellum. Neuroscience 162:656-665.

Cull-Candy SG, Usowicz MM (1987) Multiple-conductance channels activated by excitatory amino acids in cerebellar neurons. Nature 325:525-528.

Cull-Candy S, Kelly L, Farrant M (2006) Regulation of Ca2+-permeable AMPA receptors: synaptic plasticity and beyond. Curr Opin Neurobiol 16:288-297.

Derkach V, Barria A, Soderling TR (1999) Ca2+/calmodulin-kinase II enhances channel conductance of alpha-amino-3-hydroxy-5-methyl-4isoxazolepropionate type glutamate receptors. Proc Natl Acad Sci U S A 96:3269-3274

Fucile S, Miledi R, Eusebi F (2006) Effects of cyclothiazide on GluR1/AMPA receptors. Proc Natl Acad Sci U S A 103:2943-2947.

Gebhardt C, Cull-Candy SG (2006) Influence of agonist concentration on AMPA and kainate channels in CA1 pyramidal cells in rat hippocampal slices. J Physiol 573:371-394.

Greger IH, Ziff EB, Penn AC (2007) Molecular determinants of AMPA receptor subunit assembly. Trends Neurosci 30:407-416.

Herlitze S, Raditsch M, Ruppersberg JP, Jahn W, Monyer H, Schoepfer R, Witzemann V (1993) Argiotoxin detects molecular differences in AMPA receptor channels. Neuron 10:1131-1140.

Hollmann M, Hartley M, Heinemann S (1991) Ca2+ permeability of KAAMPA-gated glutamate receptor channels depends on subunit composition. Science 252:851-853.

Isaac JT, Ashby M, McBain CJ (2007) The role of the GluR2 subunit in AMPA receptor function and synaptic plasticity. Neuron 54:859-871.

Jackson AC, Nicoll RA (2011a) Stargazin (TARP gamma-2) is required for compartment-specific AMPA receptor trafficking and synaptic plasticity in cerebellar stellate cells. J Neurosci 31:3939-3952.

Jackson AC, Nicoll RA (2011b) The expanding social network of ionotropic glutamate receptors: TARPs and other transmembrane auxiliary subunits. Neuron 70:178-199.

Jahr CE, Stevens CF (1987) Glutamate activates multiple single channel conductances in hippocampal neurons. Nature 325:522-525.

Jin R, Banke TG, Mayer ML, Traynelis SF, Gouaux E (2003) Structural basis for partial agonist action at ionotropic glutamate receptors. Nat Neurosci 6:803-810.

Jonas P, Major G, Sakmann B (1993) Quantal components of unitary EPSCs at the mossy fibre synapse on CA3 pyramidal cell of rat hippocampus. J Physiol 472:615-663.

Kamboj SK, Swanson GT, Cull-Candy SG (1995) Intracellular spermine confers rectification on rat calcium-permeable AMPA and kainate receptors. J Physiol 486:297-303.

Kato AS, Zhou W, Milstein AD, Knierman MD, Siuda ER, Dotzlaf JE, Yu H, Hale JE, Nisenbaum ES, Nicoll RA, Bredt DS (2007) New transmembrane AMPA receptor regulatory protein isoform, gamma-7, differentially regulates AMPA receptors. J Neurosci 27:4969-4977.

Kato AS, Siuda ER, Nisenbaum ES, Bredt DS (2008) AMPA receptor subunit-specific regulation by a distinct family of type II TARPs. Neuron 59:986-996.

Kim KS, Yan D, Tomita S (2010) Assembly and stoichiometry of the AMPA receptor and transmembrane AMPA receptor regulatory protein complex. J Neurosci 30:1064-1072.

Klugbauer N, Dai S, Specht V, Lacinová L, Marais E, Bohn G, Hofmann F (2000) A family of gamma-like calcium channel subunits. FEBS Lett 470:189-197. 
Koh DS, Burnashev N, Jonas P (1995) Block of native Ca(2+)-permeable AMPA receptors in rat brain by intracellular polyamines generates double rectification. J Physiol 486:305-312.

Kott C, Sager C, Tapken D, Werner M, Hollmann M (2009) Comparative analysis of the pharmacology of GluR1 in complex with transmembrane AMPA receptor regulatory proteins $\gamma 2, \gamma 3, \gamma 4$ and $\gamma 8$. Neuroscience 158:77-88.

Kromann H, Krikstolaityte S, Andersen AJ, Andersen K, Krogsgaard-Larsen P, Jaroszewski JW, Egebjerg J, Strømgaard K (2002) Solid-phase synthesis of polyamine toxin analogues: potent and selective antagonists of Ca2+-permeable AMPA receptors. J Med Chem 45:5745-5754.

Lu W, Shi Y, Jackson AC, Bjorgan K, During MJ, Sprengel R, Seeburg PH, Nicoll RA (2009) Subunit composition of synaptic AMPA receptors revealed by a single-cell genetic approach. Neuron 62:254-268.

Malinow R, Malenka RC (2002) AMPA receptor trafficking and synaptic plasticity. Annu Rev Neurosci 25:103-126.

Menuz K, Stroud RM, Nicoll RA, Hays FA (2007) TARP auxiliary subunits switch AMPA receptor antagonists into partial agonists. Science 318:815-817.

Milstein AD, Nicoll RA (2008) Regulation of AMPA receptor gating and pharmacology by TARP auxiliary subunits. Trends Pharmacol Sci 29:333-339.

Milstein AD, Nicoll RA (2009) TARP modulation of synaptic AMPA receptor trafficking and gating depends on multiple intracellular domains. Proc Natl Acad Sci U S A 27:11348-11351.

Milstein AD, Zhou W, Karimzadegan S, Bredt DS, Nicoll RA (2007) TARP subtypes differentially and dose-dependently control synaptic AMPA receptor gating. Neuron 55:905-918.

Mosbacher J, Schoepfer R, Monyer H, Burnashev N, Seeburg PH, Ruppersberg JP (1994) A molecular determinant for submillisecond desensitization in glutamate receptors. Science 266:1059-1062.

Nicoll RA, Tomita S, Bredt DS (2006) Auxiliary subunits assist AMPA-type glutamate receptors. Science 311:1253-1256.

Nilsen A, England PM (2007) A subtype-selective, use-dependent inhibitor of native AMPA receptors. J Am Chem Soc 129:4902-4903.

Panchenko VA, Glasser CR, Partin KM, Mayer ML (1999) Amino acid substitutions in the pore of rat glutamate receptors at sites influencing block by polyamines. J Physiol 520:337-357.

Partin KM, Bowie D, Mayer ML (1995) Structural determinants of allosteric regulation in alternatively spliced AMPA receptors. Neuron 14:833-843.

Priel A, Selak S, Lerma J, Stern-Bach Y (2006) Block of kainate receptor desensitization uncovers a key trafficking checkpoint. Neuron 52:1037-1046.

Prieto ML, Wollmuth LP (2010) Gating modes in AMPA receptors. J Neurosci 30:4449-4459.

Robinson HP, Sahara Y, Kawai N (1991) Nonstationary fluctuation analysis and direct resolution of single channel currents at postsynaptic sites. Biophysical J 59:295-304.

Rosenmund C, Stern-Bach Y, Stevens SF (1998) The tetrameric structure of a glutamate receptor channel. Science 280:1596-1599.

Sager C, Tapken D, Kott S, Hollmann M (2009) Functional modulation of AMPA receptors by transmembrane AMPA receptor regulatory proteins. Neuroscience 158:45-54.

Shi Y, Lu W, Milstein AD, Nicoll RA (2009) The stoichiometry of AMPA receptors and TARPs varies by neuronal cell type. Neuron 62:633-640.

Sigworth FJ (1980) The variance of sodium current fluctuations at the node of Ranvier. J Physiol 307:97-129.

Smith TC, Howe JR (2000) Concentration-dependent substate behavior of native AMPA receptors. Nat Neurosci 3:992-997.
Sobolevsky AI, Rosconi MP, Gouaux E (2009) X-ray structure, symmetry and mechanism of an AMPA-subtype glutamate receptor. Nature 462: 745-756.

Sommer B, Kohler M, Sprengel R, Seeburg PH (1991) RNA editing in brain controls a determinant of ion flow in glutamate-gated channels. Cell 67:11-19.

Soto D, Coombs ID, Kelly L, Farrant M, Cull-Candy SG (2007) Stargazin attenuates intracellular polyamine block of calcium-permeable AMPA receptors. Nat Neurosci 10:1260-1267.

Soto D, Coombs ID, Renzi M, Zonouzi M, Farrant M, Cull-Candy SG (2009) Selective regulation of long-form calcium-permeable AMPA receptors by an atypical TARP, gamma-5. Nat Neurosci 12:277-285.

Strømgaard K, Mellor I (2004) AMPA receptor ligands: synthetic and pharmacological studies of polyamines and polyamine toxins. Med Res Rev 24:589-620.

Strømgaard K, Jensen LS, Vogensen SB (2005) Polyamine toxins: development of selective ligands for ionotropic receptors. Toxicon 45:249-254.

Suzuki E, Kessler M, Arai AC (2008) The fast kinetics of AMPA GluR3 receptors is selectively modulated by the TARPs gamma 4 and gamma 8 . Mol Cell Neurosci 38:117-123.

Swanson GT, Kamboj SK, Cull-Candy SG (1997) Single-channel properties of recombinant AMPA receptors depend on RNA editing, splice variation, and subunit composition. J Neurosci 17:58-69.

Tikhonov DB (2007) Ion channels of glutamate receptors: structural modeling. Mol Membr Biol 24:135-147.

Tomita S, Chen L, Kawasaki Y, Petralia RS, Wenthold RJ, Nicoll RA, Bredt DS (2003) Functional studies and distribution define a family of transmembrane AMPA receptor regulatory proteins. J Cell Biol 161:805-816.

Tomita S, Adesnik H, Sekiguchi M, Zhang W, Wada K, Howe JR, Nicoll RA, Bredt DS (2005) Stargazin modulates AMPA receptor gating and trafficking by distinct domains. Nature 435:1052-1058.

Toth K, McBain CJ (1998) Afferent-specific innervation of two distinct AMPA receptor subtypes on single hippocampal interneurons. Nat Neurosci 1:572-578.

Traynelis SF, Silver RA, Cull-Candy SG (1993) Estimated conductance of glutamate receptor channels activated during EPSCs at the cerebellar mossy fiber-granule cell synapse. Neuron 11:279-289.

Traynelis SF, Wollmuth LP, McBain CJ, Menniti FS, Vance KM, Ogden KK, Hansen KB, Yuan H, Myers SJ, Dingledine R, Sibley D (2010) Glutamate receptor ion channels: structure, regulation, and function. Pharmacol Rev 62:405-496.

Turetsky D, Garringer E, Patneau DK (2005) Stargazin modulates native AMPA receptor functional properties by two distinct mechanisms. J Neurosci 25:7438-7448.

Washburn MS, Dingledine R (1996) Block of alpha-amino-3-hydroxy-5methyl-4-isoxazolepropionic acid (AMPA) receptors by polyamines and polyamine toxins. J Pharmacol Exp Ther 278:669-678.

Washburn MS, Numberger M, Zhang S, Dingledine R (1997) Differential dependence on GluR2 expression of three characteristic features of AMPA receptors. J Neurosci 17:9393-9406.

Zhang W, Robert A, Vogensen SB, Howe JR (2006) The relationship between agonist potency and AMPA receptor kinetics. Biophys J 91:1336-1346.

Zhang W, Cho Y, Lolis E, Howe JR (2008) Structural and single-channel results indicate that the rates of ligand binding domain closing and opening directly impact AMPA receptor gating. J Neurosci 28:932-943.

Ziff EB (2007) TARPs and the AMPA receptor trafficking paradox. Neuron 53:627-633. 